\title{
Hommage à Laurent WAHL (1967-2008)
}

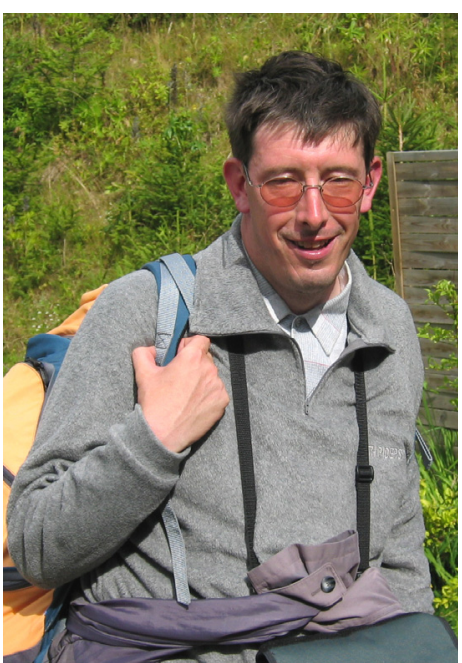

Laurent WAHL nous a quitté brutalement le 18 décembre 2008 dans la pleine force de l'âge. Rien ne laissait prévoir l'accident de santé qui l'a brutalement emporté à l'âge de 41 ans, alors qu'il évoquait encore la veille ses projets de recherche et de voyage pour les vacances de fin d'année.

Laurent WAHL a effectué l'ensemble de ses études supérieures à la Faculté de Géographie de Strasbourg. Dès la seconde année, il révélait déjà fortement sa passion du terrain, de la climatologie et de la météorologie, ainsi qu'un sens aigu de l'observation du temps sensible. Ses connaissances exceptionnelles et précises de la géographie physique et humaine des Vosges, de la Forêt Noire et du Fossé rhénan ont été le fruit d'observations et d'analyse des paysages glanées de centaines de randonnées effectuées en toute saisons depuis son enfance.

Après la soutenance de son mémoire de maîtrise particulièrement bien documenté 'Etude des déterminants climatiques vis-à-vis de la qualité du moût et du vin en Alsace de 1945 à 1990', il soutint un mémoire de DEA en 1991 'Contribution à l'étude de l'évolution des brouillards dans le Fossé rhénan entre le matin et l'après-midi à l'aide des données NOAA$A V H R R$ '. Ce travail original a permis la mise en place de méthodes supervisées, intégrant des contrôles sur le terrain et une confrontation systématique avec les paramètres climatiques de surface et d'altitude Ses résultats novateurs avaient fait l'objet d'un long exposé en allemand, vivement apprécié, aux journées du Klima Arbeitskreis (réunions annuelles des géographes climatologues allemands) en juin 1992 à Flensburg. C'est pourquoi, l'idée est venue d'engager une thèse dans le cadre du programme climatologique franco-germano-suisse REKLIP, intitulée 'Etude de la dynamique spatio-temporelle des brouillards au sein du Fossé rhénan méridional à l'aide d'images NOAA-AVHRR', soutenue en 1997.

Ses centres d'intérêt en climatologie se sont ensuite diversifiés : entre autres, analyse des tempêtes de décembre 1999, de la canicule de 2003, recherches sur l'évolution des névés temporaires et les risques d'avalanches dans les moyennes montagnes d'Europe centrale dans le cadre de plusieurs collaborations, notamment l'équipe COSTEL à Rennes, à laquelle il venait de s'associer dans le cadre du GDR 2663 CNRS RICLIM (Risques liés au climat). Et il prévoyait de participer activement au XXII ${ }^{\text {ème }}$ colloque de climatologie de l'AIC à Cluj et d'y présenter une communication conjointe avec Olivier PLANCHON.

Excellent pédagogue, passionné et très disponible, Laurent WAHL a été recruté maître de conférences au Département de Géographie de l'Université de Nancy en 1999. II y était unanimement apprécié par sa grande disponibilité, sa gentillesse et son implication dans des responsabilités collectives et les nombreuses sorties de terrain qu'il organisait en Lorraine, dans le massif vosgien, en Allemagne et en Autriche. II savait remarquablement bien transmettre avec douceur son savoir-faire et ses connaissances, et encadrait avec efficacité des mémoires de maîtrise. Au total, Laurent, collègue attachant et compétent, nous manquera beaucoup au sein de la communauté des climatologues géographes francophones. 